\title{
Radiological Assessment of Post Surfactant Changes in Respiratory Distress Syndrome
}

\author{
Sunny Mishra ${ }^{1}$, Pratik Tailor ${ }^{2}$, Mona Shastri ${ }^{3}$ \\ ${ }^{1}$ Resident, Department of Radiodiagnosis, SMIMER Medical College, Surat, 7405455640 \\ ${ }^{2}$ Resident, Department of Radiodiagnosis, SMIMER Medical College, Surat, 9974334242 \\ ${ }^{3}$ Professor \& Head, Department of Radiology, SMIMER Medical College, Surat \\ (E-301, PG Boys Hostel, SMIMER Medical College campus, Surat - 395010)
}

\begin{abstract}
Seventy three preterm neonates with clinical and radiological evidence of typical RDS having median gestational age 30 weeks and median birth weight $1240 \mathrm{~g}$ were studied to evaluate the radiological changes after therapeutic use of surfactant and the value of ultrasound in its screening. The chest radiographs were taken before and 72 hours post surfactant treatment with surfactant application ranging from $1 \mathrm{~h}$ to $18 \mathrm{~h}$ after birth. $72 \%$ cases revealed uniform or asymmetrical extension of pulmonary aeration. Asymmetrical improvement (29\%) was seen on the left side right side and usually disappeared within two to five days. 5 neonates (13.5\%) showed no change, of which 2 succumbed to death.
\end{abstract}

Keywords: RDS, surfactant, HMD, X-rays

\section{Introduction}

Respiratory distress syndrome is principally a malady of premature infants born before 32 weeks of gestation. The treatment for RDS consists of Oxygen therapy along with surfactant supplementation.Radiography has been the longestablished technique in the identification and follow-up of children suffering from RDS. The core pathogenesis involves developmental immaturity of the lungs, leading to the deficiency of pulmonary surfactant. The peril of developing RDS decreases with both increasing gestational age and birth weight. The incidence rate is $80 \%$ in infants $<28$ weeks' gestation, $60 \%$ at 29 weeks, $15-30 \%$ at $32-34$ weeks, and declines with maturity to $5 \%$ at $35-36$ weeks. Accordingly, the RDS incidence rate is estimated to be $80 \%$ for infants weighing $<750 \mathrm{~g}$ at birth and $55 \%$ for infants weighing 750-1000 g. However, in recent years, with the application of antenatal corticosteroids and delivery room pulmonary surfactant, both typical and severe RDS in premature infants have greatly declined. Greater awareness of RDS has led to a more frequent diagnosis in term neonates. We have also found that RDS in term infants accounts for $3.6 \%$ of all term in-patients. The typical chest radiography findings post surfactant include (1) uniform improvement, (2) asymmetrical improvement, (3) no improvement or (4) interstitial emphysema - after therapeutic use of surfactant

\section{Aims \& Objectives}

The aim of the following study was to determine typical pulmonary X-ray findings after therapeutic use of surfactant and to analyze their clinical significance.

\section{Materials And Methods}

The study was conducted at a tertiary care hospital of south Gujarat from December 2014 to July 2015 to evaluate the influence of surfactant administration on radiographic appearance of 73 neonates with typical clinical and radiological diagnosis of RDS were selected, for whom adequate chest radiographs from before and $72 \mathrm{~h}$ after treatment were available. Infants with a gestational age (GA) $<34$ weeks with need for assisted ventilation were included. All infants with clinical evidence of congenital pneumonia or sepsis were excluded in order to prevent mixed effects between changes caused by surfactant application and changes of infectious origin.

The surfactant preparation used SURVANTA ${ }^{\circledR}$ (beractantintratracheal suspension)a liquid solution extracted from bovine lungs. The surfactant solution was instilled via a small-sized nasogastric tube, which was inserted into the endotracheal tube with the tip just above the carina. Depending on the clinical situation, up to four applications were allowed, each bolus containing $50 \mathrm{mg}$ dry substance per kg body weight.

Number of doses depends on the clinical situation.Median birth weight was $1025 \mathrm{~g}$ (range 650-1510 g) and median gestational age (GA) was 28 weeks (range 25-34 weeks). Pre-surfactant instillation RDS severity was graded from I to IV. Pretreatment radiographs were obtained between 40 mins to $20 \mathrm{~h}$ (median $3 \mathrm{~h}$ ) after birth. The time of surfactant application ranged from 1 to $12 \mathrm{~h}$ (median $6.5 \mathrm{~h}$ ) after birth. Timing of post-treatment radiographs was based on the clinical situation, the first being taken usually 4-6 h after surfactant replacement. Both pretreatment and the first posttreatment chest radiographs were compared and grouped according to extent and distribution of radiographic improvement of aeration.Radiographic findings and clinical data were statistically analyzed using the chi-squared test and Student's $t$-test.

\section{Results}

Pre-surfactant instillation radiographs demonstrated severe RDS (grade III or IV) in 79 of our patients. There was a 


\section{International Journal of Science and Research (IJSR) \\ ISSN (Online): 2319-7064 \\ Index Copernicus Value (2015): 78.96 | Impact Factor (2015): 6.391}

good correlation between severity of RDS seen in pretreatment chest radiographs and pretreatment $\mathrm{FiO}_{2}(P<$ $0.01)$. Alterations in pulmonary aeration were classified into four categories: (1) uniform improvement, (2) asymmetrical improvement, (3) no improvement and (4) interstitial emphysema. The most common findings after surfactant instillation were uniform $(n=27)$ or asymmetrical $(n=23)$ improvement of aeration of the lungs.

\subsection{Uniform Improvement}

The extent of uniform improvement of pulmonary aeration after surfactant dispensation was very capricious. Most of these patients (21/27) showed almost Complete normalisation of lung aeration with a single surfactant dose was noticed in majority of the patients(21/27patients; $77 \%$ ). In 5 of themfew discrete residual streaky radioopacities remained. In the remaining 6 patients presurfactant radioopacity still prevailed after a single dose of surfactant. However these patients showed normal radiographs after second dose of surfactant. Only one case of pneumothorax (PTX) was noted and severe intracranial haemorrhage was found in 3 patients. A high survival rate was seen (95\%) with 19 of these 26 infants had spontaneously breathing at day 28 of life without the need for supplementary oxygen.

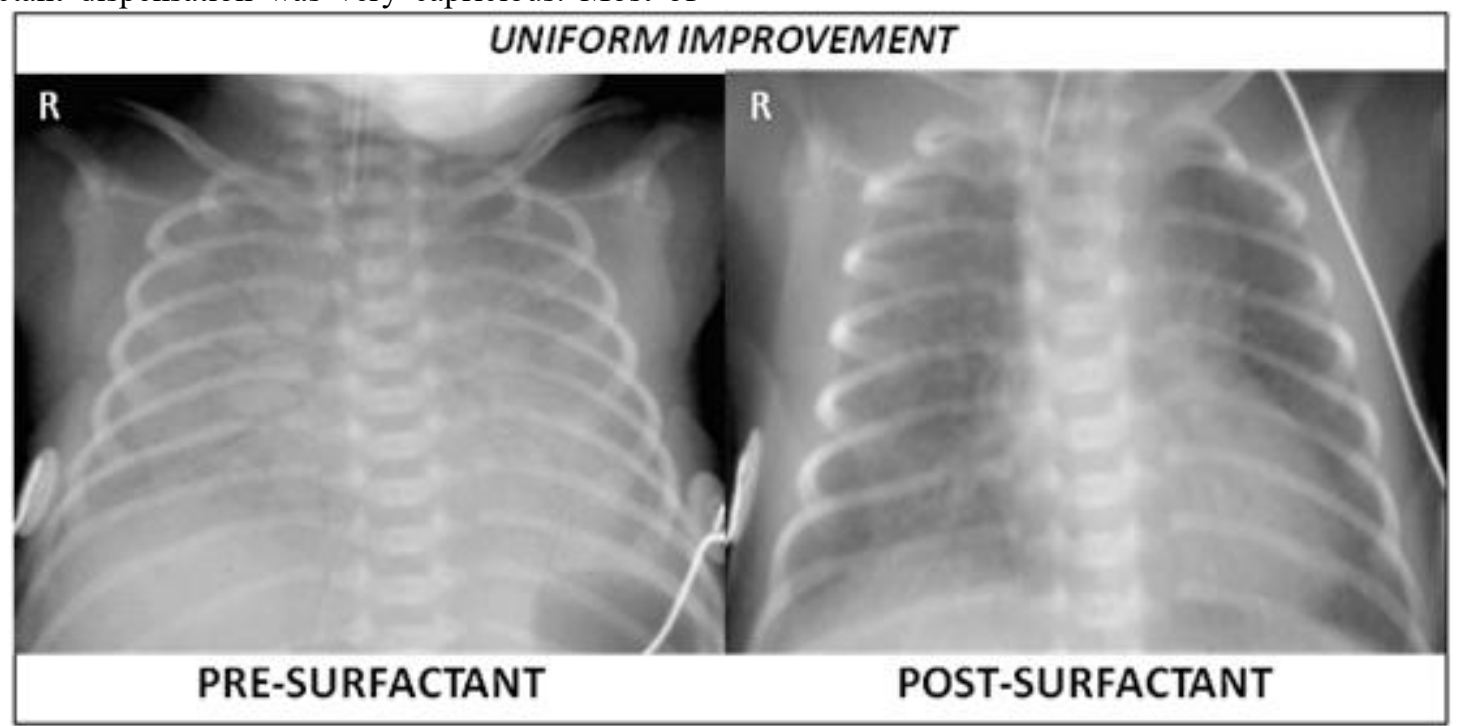

Figure 1: 28-week premature infant shows diffuse respiratory distress syndrome. Post surfactant lungs show uniform improvement with complete normalisation of lung aeration after a single surfactant dose

\subsection{Asymmetrical/focal improvement}

Patchy clearance or Asymmetrical/focal improvement of aeration was often more marked in the central or upper regions of the right lung. These findings vanished within a few days, frequently after instillation of a retreatment dose of surfactant (17/23 patients). In three patients malpositionedendotracheal tube was noted with the bevel positioned just above the carina was noted.We also observed that a small nasogastric tube is used for the instillation technique may lead to under-delivery of the surfactant and result in patchy clearance. There was a higher incidence of pneumothorax $(2 / 23)$, intracranial haemorrhage $(3 / 23)$ and mortality $(2 / 23)$ as compared to the patients with uniform improvement however these observations were statistically insignificant.
A reduced oxygen requirement was noted in both uniform and asymmetrical clearance types. There were no statistically significant differences in gestational age, birth weight or severity of RDS between the two groups. both groups showed higher incidence of Antenatal administration of corticosteroids but there was no statistically significant difference as compared to the patients with no improvement or interstitial emphysema.

\subsection{No improvement}

Post-surfactant chest radiographs with no obvious changes in the aeration was observed in nine patients. Subsequent surfactant applications were absolutely ineffective. Majority of these patients $(8 / 9)$ had to be instilled with surfactant more than once, with a maximum frequency of repetition being 4 times. 


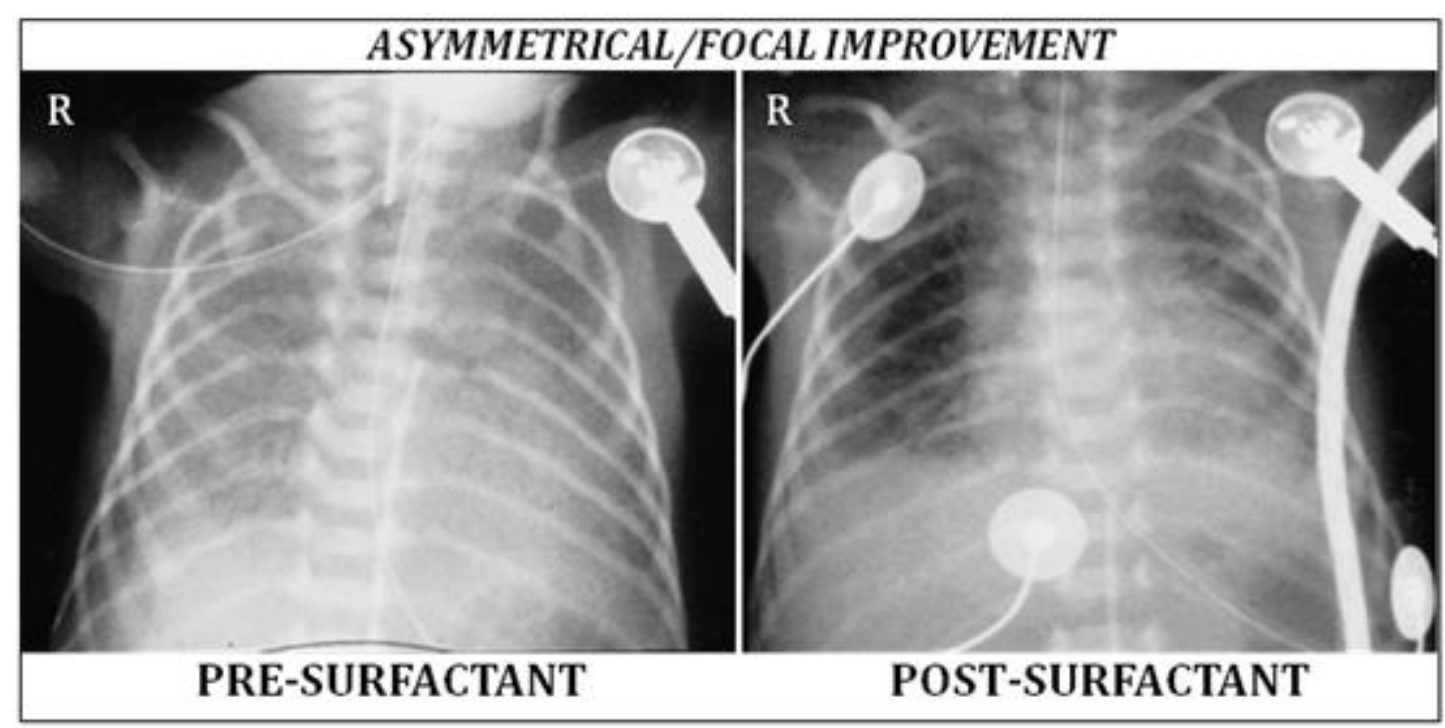

Figure 2: Preterm infant with (GA 28 weeks, 1100g) with severe RDS: chest radiographs with typical severe RDS a before and $\mathbf{b}$ after surfactant treatment. Clearing is more pronounced in the central regions

\subsection{Interstitial emphysema}

Amongst the grave prognostic signs of deterioration of RDS post surfactant, development of pulmonary Interstitial emphysema (PIE) is a major finding. Most of the patients developed PTX (4/13) and severe intracranial haemorrhage $(8 / 13) ; 73 \%$ of the infants with PIE after surfactant treatment $(5 / 13)$ died within the 1-month of life, compared with $8.3 \%$ $(5 / 60)$ in the groups with initial improvement of aeration on the first post treatment chest radiograph. Eight of the surving patients were ventilated mechanically for a stretched period (3 weeks or more). The other three group-surviving patients showed no clear correlation between the post surfactant radiographic response and the period of mechanical ventilation. Birth weight and gestational age were the best clinical parameters predicting long-term outcome survival, duration of artificial ventilation and development of intracranial haemorrhage.

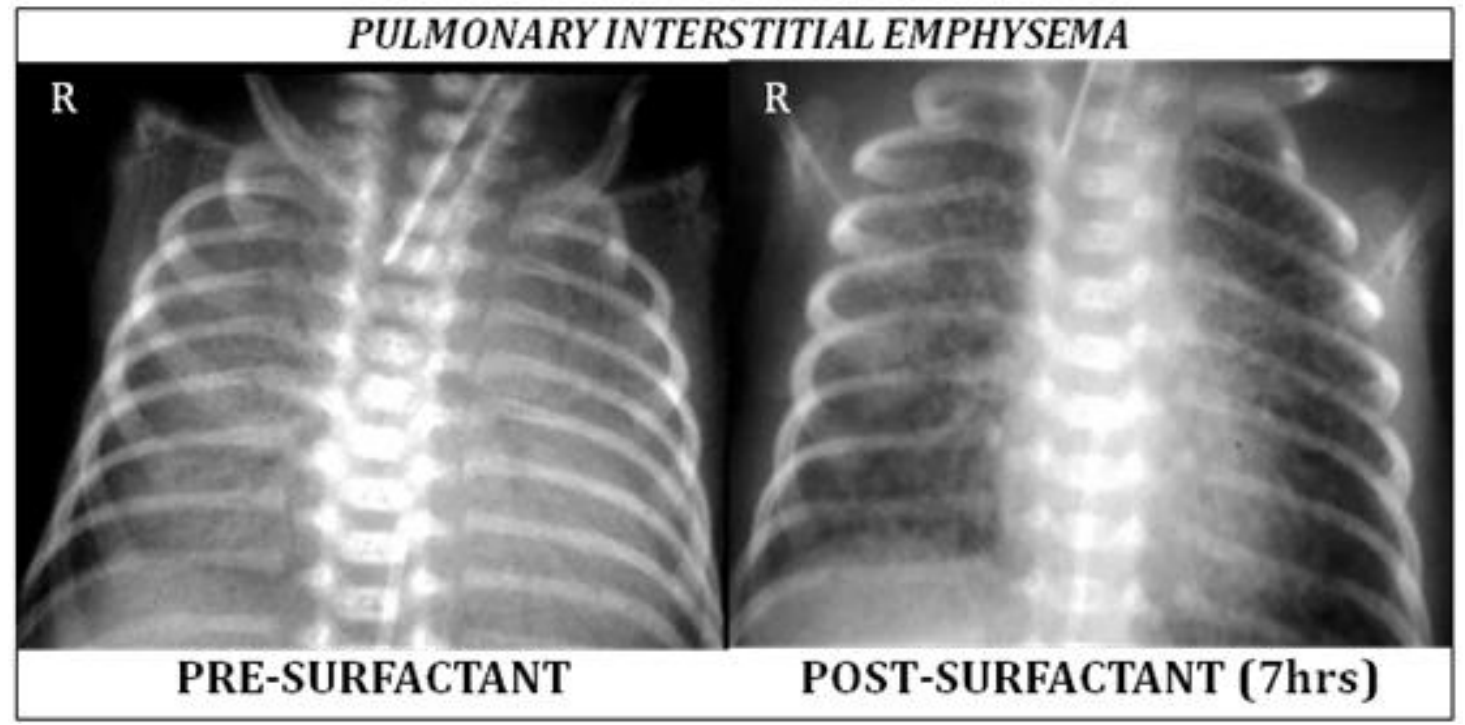

Figure 3: 27-week premature infant shows severe diffuse respiratory distress syndrome. At 7 hours, the lungs are over expanded with multiple linear areas of lucency, indicating pulmonary interstitial emphysema

\section{Discussion}

The efficacy of surfactant instillation in infants with RDS is undeniable and has been publicized by numerous studies. Clinical progress isconvoyed by remarkable improvement of aeration of the lung on post-treatment chest radiographs seen in 37 out of our 73 study patients $(50.6 \%)$. Similar results were published by $\operatorname{Bick}$ et $\operatorname{al}^{9}(35 \%, 46 / 130)$. In contrast, Clarke et $\mathrm{al}^{10}$ found no evidence of RDS on post-treatment radiographs in $49 \%$ of 80 patients treated prophylactically with surfactant. Asymmetrical clearing has been reported by several authors - Clarke et $\mathrm{al}^{10}$ found asymmetrical or central clearing in $22 \%$ of 80 infants treated prophylactically with surfactant and Bick et al ${ }^{11}$ found an incidence of $35 \%$ in a study group of 130 patients treated therapeutically with surfactant. The incidence in our study group was similar (32\%). In summary, about $70 \%$ of infants with RDS treated with surfactant $(69 \%$ in our study, $69 \%$ in the study of Bick et $\mathrm{al}^{9} 71 \%$ in the study of Clarke et al ${ }^{10}$ showed homogeneous or asymmetrical improvement of aeration with rapid reduction of oxygen requirement after surfactant treatment. As expected, patients with uniform 


\section{International Journal of Science and Research (IJSR) \\ ISSN (Online): 2319-7064}

Index Copernicus Value (2015): 78.96 | Impact Factor (2015): 6.391

clearing had the best long-term outcome: 47 (94\%) of our 50 patients survived, and 32 (68\%) of our surviving infants were breathing spontaneously at day 28 of life without the need for supplementary oxygen. Disproportionate aeration usually resolved within 3-5 days, often after retreatment with surfactant ${ }^{8}$.

We speculate that asymmetrical improvement of aeration after surfactant treatment might be the result of maldistribution ${ }^{10}$ Maldistribution due to a malpositionedendotracheal tube was seen. Focal clearing might be due to administration of an insufficient amount of surfactant, making further applications necessary. Often the dependent regions of the lungs are less ventilated ${ }^{11}$. Thus, selective aeration of the anterior and upper regions of the lungs before surfactant replacement might explain the picture of central clearing seen after surfactant replacement ${ }^{5}$. It is still possible that interstitial emphysema developed during the time between the pretreatment radiograph and the initiation of surfactant replacement. Bronchiolar epithelial disruption may cause release of plasma proteins into the airways, as well as leakage of surfactant into the interstitium, and might prevent success of surfactant treatment. ${ }^{6}$ Both release of plasma proteins and leakage of surfactant might explain the cases of unusually severe interstitial emphysema after surfactant treatment. ${ }^{3}$

\section{Conclusion}

Our results show that: (1) about 38\% of infants with RDS show complete improvement after surfactant treatment with good correlation to clinical data; (2) asymmetrical or focal air disturbances are frequent (34\%), but usually resolve after retreatment with surfactant without causing any significant clinical problems; and (3) development of interstitial emphysema after surfactant treatment is a grave prognostic sign, emphasizing the sensitivity of the immature lung to barotrauma. Therefore, early chest radiographic monitoring of the therapeutic effect of surfactant, especially when retreatment doses of surfactant are given, might prevent over-inflation and help in setting appropriate ventilator parameters, improving outcome.

\section{References}

[1] Gortner, L., Bartmann, P., Pohlandt, F., Bernsau, U., Porz, F., Hellwege, H.\&Hentschel, R. (1992). Early treatment of respiratory distress syndrome with bovine surfactant in very preterm infants: a multicenter controlled clinical trial. Pediatric pulmonology, 14(1), 4-9.

[2] Halliday, H. L., Tarnow-Mordi, W. O., Corcoran, J. D., \& Patterson, C. C. (1993). Multicentre randomised trial comparing high and low dose surfactant regimens for the treatment of respiratory distress syndrome (the Curosurf 4 trial). Archives of disease in childhood, 69(3 Spec No), 276-280.

[3] Levine, D., Edwards 3rd, D. K., \& Merritt, T. A. (1991). Synthetic vs human surfactants in the treatment of respiratory distress syndrome: radiographic findings. AJR. American journal of roentgenology, 157(2), 371374.
[4] Charon, A., Taeusch, H. W., Fitzgibbon, C., Smith, G. B., Treves, S. T., \& Phelps, D. S. (1989). Factors associated with surfactant treatment response in infants with severe respiratory distress syndrome. Pediatrics, 83(3), 348-354.

[5] Zecca, E., De Luca, D., Marras, M., Caruso, A., Bernardini, T., \&Romagnoli, C. (2006). Intrahepaticcholestasis of pregnancy and neonatal respiratory distress syndrome. Pediatrics, 117(5), 1669 1672.

[6] Heneghan, M. A., Sosulski, R., \& Alarcon, M. B. (1987). Early pulmonary interstitial emphysema in the newborn: a grave prognostic sign. Clinical pediatrics, 26(7), 361-365.

[7] Soll, R. F., Horbar, J. D., Griscom, N. T., Barth, R. A., Lucey, J. F., \&Taeusch, H. W. (1991). Radiographic findings associated with surfactant treatment. American journal of perinatology, 8(02), 114-118.

[8] Edwards, D. K., Hilton, S. V., Merritt, T. A., Hallman, M., Mannino, F., \& Boynton, B. R. (1985). Respiratory distress syndrome treated with human surfactant: radiographic findings. Radiology, 157(2), 329-334.

[9] Bick, U., Müller-Leisse, C., Tröger, J., Jorch, G., Roos, N., zuWendischhoff, J. M., ... \&Hörnchen, H. (1992). Therapeutic use of surfactant in neonatal respiratory distress syndrome. Pediatric radiology, 22(3), 169-173.

[10] Clarke, E. A., Siegle, R. L., \& Gong, A. K. (1989). Findings on chest radiographs after prophylactic pulmonary surfactant treatment of premature infants. American Journal of Roentgenology, 153(4), 799-802.

[11] Konishi, M., Fujiwara, T., Chida, S., Maeta, H., Shimada, S., Kasai, T., \& Murakami, Y. (1992). A prospective, randomized trial of early versus late administration of a single dose of surfactant-TA. Early human development, 29(1-3), 275-282.

[12] Reilly, B. J. (1975). Neonatal radiology. Regional distribution of atelectasis and fluid in the neonate with respiratory distress. Radiologic clinics of North America, 13(2), 225-250. 